\title{
An Approach of Electronic Subdividing Method for Measuring Straightness and Displacement of a Precision Linear Stage Simultaneously
}

\author{
Hsiu-An Tsai ${ }^{1,2}$ and Shao-Ching Chen ${ }^{1}$ \\ ${ }^{1}$ Department of Industrial Upgrading Service, Metal Industries Research \& Development Centre, Kaohsiung, Taiwan \\ ${ }^{2}$ Department of Mechanical Engineering, National Cheng Kung University, Tainan, Taiwan
}

\begin{abstract}
Optical encoders are commonly used in modern positioning systems. The accuracy and resolution of the optical encoders are always restricted by generated sinusoidal signals and the assembly technique. In this study, an electronic signal subdividing system is developed. This system is based on FPGA in combination with A/D and D/A converting circuits. Subdividing algorithm improves the segmenting signal amplitude method. Furthermore, we also construct a laser encoder for measuring straightness error and displacement of a linear stage simultaneously. The laser encoder consisting of the stainless steel bar and the sensor are developed for two-axis (X-and Z-axis) position measurement. The two dimensional sinusoidal array on the stainless steel bar are machined by ultrasonic elliptical vibration cutting system. The stainless steel bar has a three dimensional micro-structured surface, which is a superposition of periodic sinusoidal waves in the X- and Z-directions with spatial wavelengths of $350 \mu \mathrm{m}$ and amplitudes of $0.5 \mu \mathrm{m}$. The laser-based two-axis position sensor is used to detect local slope profiles of the grid surface, and the displacement and straightness error could be extracted from the X-and Z-axis sensing signal. The sensing signal is processed by FPGA subdividing system. In addition, the proposed subdividing method is verified by the performances and effects of measuring results.
\end{abstract}

\section{Introduction}

Precision metrology is still playing an important role in precision on-machine measurement, positioning and manufacturing from the point of a wide range of measurements with high accuracy and resolution [1]-[8].

With the rapid progress in the field of nanotechnology, there is high demand for new displacement measurement technologies to be able to achieve sub-nanometer level resolution with long-term reliability. In accordance with the increasing market demand for ultra-precision technology, a high precision multi-degree-of-freedom displacement measurement technology has become important for industrial applications such as the field of manufacturing and inspection because physical quantities, such as linear and angular displacements, are key parameters for keeping and improving quality control of a production system [1], [2], [5]-[8].

Linear stages are commonly used in machine tools, semiconductor instruments, precision assembly processing and nanometer level positioning [1]. In addition, high accuracy stages have commonly been used in semiconductor processing, PCB drilling processing, micromachining processing, precision assembly processing, inspection processing, etc. Any positioning stage is considered to have six-degrees-of-freedom. There errors can be defined as occurring along the reference coordinate system.

It should be noticed that the term "straightness error" is generally used to refer to many aspects of engineering quality, such as work-piece straightness, motion straightness, etc. Optical measurements of straightness errors have been widely used in the field of engineering metrology. The straightness datum that can be measured by means of various optical accessories such as: autocollimators, alignment telescopes and optical theodolites are commonly adopted. Another method employs a laser beam to generate a reference line, which has the properties of small divergence and high intensity. Moreover, many other methods were developed with high alignment accuracy, such as the polarimetry method [9], the polarizing method [10], the optical compensation method, and the Zeeman laser interferometer method [11]. However, the mentioned measurement devices are not suitable to be installed on precise positioning machinery for on-line measurement as a result of heavy overall structure. Recently, a laser diode was adopted for straightness measurement since it is small in size and low in power [12]. Furthermore, optical encoders are commonly used in modern positioning systems. Moreover, the accuracy and resolution of the optical encoder is always restricted by generated sinusoidal signals and the assembly technique. Therefore, an 
electronic signal subdividing system is developed for measuring straightness error and displacement of a linear stage simultaneously.

\section{Measurement principles instrument configuration}

Figure 1 shows a diagram of the measurement system. Assume that the sensor is kept stationary and the twodimensional sinusoidal grating was placed in the $\mathrm{XZ}$ plane of the linear $\mathrm{X}$-axis stage.

An incident beam from the laser diode is collimated by the collimating lens. The collimated laser beam incidents to the beam-splitting device. It should be noticed that the beam-splitting device which is disposed in the light path of the incident light and comprises a beam splitter and a polarizer plate. And the beam splitter is used for guiding the incident light to pass the focus lens on the two-dimensional sinusoidal grating. The reflection light beam is reflected from the twodimensional sinusoidal grating and reflected by the reflective mirror to pass the focus lens on the quadrant photo diodes (QPD).

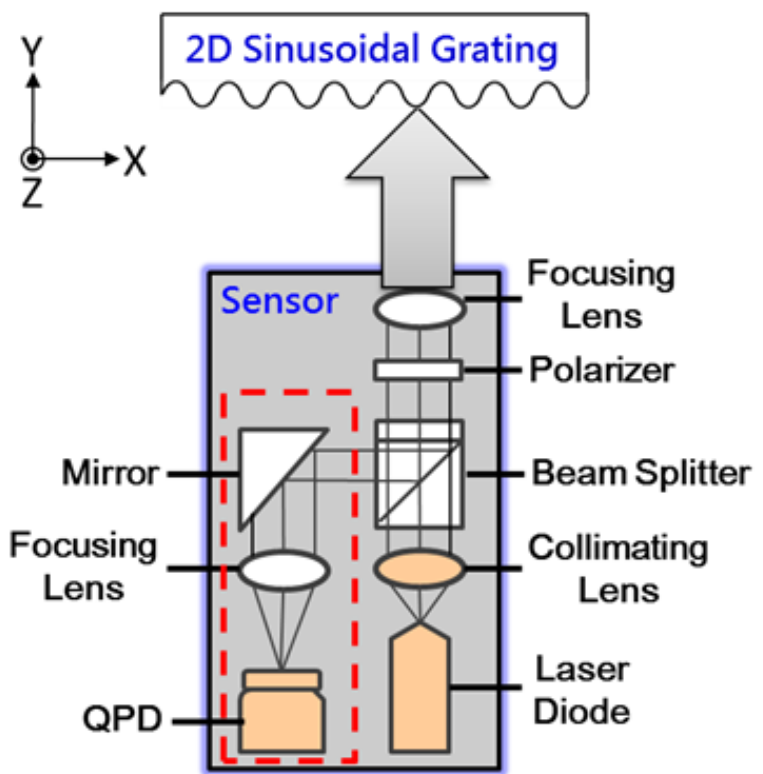

Figure 1. Configuration of the proposed measurement system.

The reflective-type two-dimensional sinusoidal grating, wherein a profile equation of the reflective-type two-dimensional sinusoidal grating is expressed as:

$$
h(x, z)=-A_{x} \cos \left(\frac{2 \pi x}{P_{x}}\right)-A_{z} \cos \left(\frac{2 \pi z}{P_{z}}\right)
$$

where $P_{x, Z}$ are the wavelengths of the reflective-type twodimensional sinusoidal grating in the $\mathrm{x}$-axis direction and the $\mathrm{z}$-axis direction. In addition, $\mathrm{A}_{\mathrm{x}, \mathrm{z}}$ are the corresponding sinusoidal amplitudes. In this study, the $\mathrm{P}_{\mathrm{x}, \mathrm{z}}$ and $\mathrm{A}_{\mathrm{x}, \mathrm{z}}$ are $350 \mu \mathrm{m}$ and $0.5 \mu \mathrm{m}$, respectively. As a result, separately performing partial differentiation to obtain the slope equations of the two-dimensional grating in the $\mathrm{X}$-axis direction and the $\mathrm{Z}$-axis direction are as shown as followed:

$$
\begin{aligned}
& m_{x}(x, z)=\frac{\partial h(x, z)}{\partial x}=\frac{2 \pi A_{x}}{P_{x}} \sin \left(\frac{2 \pi x}{P_{x}}\right) \\
& m_{z}(x, z)=\frac{\partial h(x, z)}{\partial z}=\frac{2 \pi A_{z}}{P_{z}} \sin \left(\frac{2 \pi z}{P_{z}}\right)
\end{aligned}
$$

On the other hand, we can apply the auto-collimation principle to measure $\mathrm{m}_{\mathrm{x}}(\mathrm{x}, \mathrm{z})$ and $m_{z}(x, z)$ from $\mathrm{QPD}$ :

$$
\begin{aligned}
& u=\left(\tan ^{-1} m_{x}(x, z)\right) \times 2 f \\
& v=\left(\tan ^{-1} m_{z}(x, z)\right) \times 2 f
\end{aligned}
$$

where $\mathrm{u}$ and $\mathrm{v}$ are the displacement in the $\mathrm{X}$-axis and $\mathrm{Z}$ axis directions of the QPD, respectively. And the $f$ is the focus distance from the lens to the QPD. With reference to the Eq. (4) and Eq. (5), the QPD receives the sensing signal which represents the position variation of the twodimensional sinusoidal grating in the $\mathrm{X}$ - and $\mathrm{Z}$ - directions. Therefore, an axial motion displacement volume and a radial displacement volume of the linear stage are calculated by using a slope equation and a position signal equation to calculate the sensing signals of the QPD. The $\mathrm{X}$ - axis and Z-axis motion signal equations could be expressed as:

$$
\begin{aligned}
& x=\frac{P_{x}}{2 \pi} \sin ^{-1}\left(\frac{P_{x}}{2 \pi A_{x}} \times \tan \frac{u}{2 f}\right) \\
& z=\frac{P_{z}}{2 \pi} \sin ^{-1}\left(\frac{P_{z}}{2 \pi A_{z}} \times \tan \frac{v}{2 f}\right)
\end{aligned}
$$

In other words, the $\mathrm{X}$ - axis and Z-axis motion signals are obtained as a function of the $\mathrm{u}$ and $\mathrm{v}$ values only. However, with reference to the Eq. (6) and Eq. (7), the signal discriminant analysis needs to be constructed.

In the first stage of the signal discriminant analysis procedure, the quadrant of the initial point signal $\left(\mathrm{S}_{\mathrm{i}}\right)$ is defined before knowing the sign of $\mathrm{I}_{0}, \mathrm{I}_{\mathrm{S}}$, and $\mathrm{I}_{\mathrm{C}}$ (i.e., positive or negative) are measured directly from the data acquisition products. It should be noticed that the corresponding signal output $\left(\mathrm{I}_{\mathrm{S}}\right)$ from the $\mathrm{QPD}$ was converted to a square wave afterpassing through a comparator, as shown in Fig. 2. In addition, the corresponding signal output $\left(\mathrm{I}_{\mathrm{C}}\right)$ was generated to a $90^{\circ}$ phase shift square-wave signal of the $\mathrm{I}_{\mathrm{S}}$ signal.

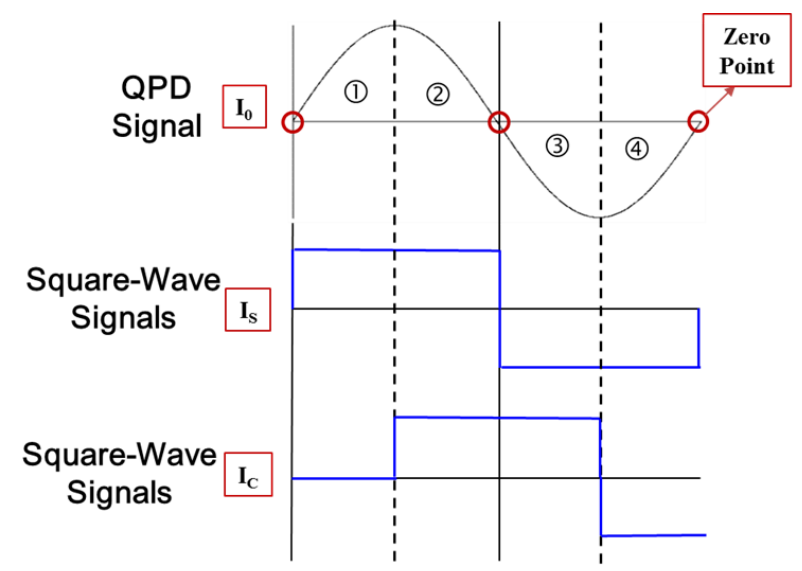

Figure 2. Scheme illustration of the corresponding signal output in measurement system. $\mathrm{I}_{0}$ : QPD output signal, $\mathrm{I}_{\mathrm{S}}$ : the corresponding signal output from the $\mathrm{QPD}$, and $\mathrm{I}_{\mathrm{C}}: 90^{\circ}$ phase shift signal output of the $\mathrm{I}_{\mathrm{S}}$ signal. 
Moreover, if the value of $\mathrm{I}_{0}>0, \mathrm{I}_{\mathrm{S}}>0$, and $\mathrm{I}_{\mathrm{C}}=0$, then the motion signal is located in the I-quadrant. Conversely, if the value of $\mathrm{I}_{0}>0, \mathrm{I}_{\mathrm{S}}>0$, and $\mathrm{I}_{\mathrm{C}}>0$, then the motion signal is located in the II-quadrant. Meanwhile, if the value of $\mathrm{I}_{0}<0, \mathrm{I}_{\mathrm{S}}<0$, and $\mathrm{I}_{\mathrm{C}}>0$, then the motion signal is located in the III-quadrant. Finally, if the value of $\mathrm{I}_{0}<0$, $\mathrm{I}_{\mathrm{S}}$ $<0$, and $\mathrm{I}_{\mathrm{C}}<0$, then the motion signal is located in the IVquadrant. Having determined the quadrant of the initial point signal, the $\mathrm{X}$ - axis and $\mathrm{Z}$-axis initial positions can be extracted from Eq. (6) and Eq. (7).

In the second stage of the signal discriminant analysis procedure, the quadrant of the terminal point signal is defined before knowing the quadrant within which the initial point lies and the number of the zero points of the sequence signal. Accordingly, we constructed the signal discriminant criterion as shown in Table 1. Having determined the quadrant of the terminal point signal, the $\mathrm{X}$ - axis and $\mathrm{Z}$-axis terminal positions can be extracted from Eq. (6) and Eq. (7).

Table 1. Signal discriminant criterion for analyzing the quadrant of the terminal point signal. $\mathrm{S}_{\mathrm{i}}$ is the initial data point of $\mathrm{I}_{0}$ and $\mathrm{K}$ is the whole numbers.

\begin{tabular}{|c|c|c|c|c|}
\hline \multirow{2}{*}{$\begin{array}{c}\text { Number of Zero Points } \\
\text { Quadrant of Initial point }\end{array}$} & \multicolumn{2}{|c|}{$2 \mathrm{~K}$} & \multicolumn{2}{c|}{$2 \mathrm{~K}+1$} \\
\hline \multirow{2}{*}{$\mathrm{I}$} & $\mathrm{S}_{\mathrm{i}}>0$ & $\mathrm{~S}_{\mathrm{i}}<0$ & $\mathrm{~S}_{\mathrm{i}}>0$ & $\mathrm{~S}_{\mathrm{i}}<0$ \\
\cline { 2 - 5 } & $\mathrm{I}$ & $\mathrm{IV}$ & $\mathrm{I}$ & $\mathrm{III}$ \\
\hline \multirow{2}{*}{$\mathrm{II}$} & $\mathrm{S}_{\mathrm{i}}>0$ & $\mathrm{~S}_{\mathrm{i}}<0$ & $\mathrm{~S}_{\mathrm{i}}>0$ & $\mathrm{~S}_{\mathrm{i}}<0$ \\
\cline { 2 - 5 } & $\mathrm{II}$ & $\mathrm{III}$ & $\mathrm{I}$ & $\mathrm{IV}$ \\
\hline \multirow{2}{*}{ III } & $\mathrm{S}_{\mathrm{i}}>0$ & $\mathrm{~S}_{\mathrm{i}}<0$ & $\mathrm{~s}_{\mathrm{i}}>0$ & $\mathrm{~S}_{\mathrm{i}}<0$ \\
\cline { 2 - 5 } & $\mathrm{II}$ & $\mathrm{III}$ & $\mathrm{I}$ & $\mathrm{IV}$ \\
\hline \multirow{2}{*}{$\mathrm{IV}$} & $\mathrm{S}_{\mathrm{i}}>0$ & $\mathrm{~S}_{\mathrm{i}}<0$ & $\mathrm{~S}_{\mathrm{i}}>0$ & $\mathrm{~S}_{\mathrm{i}}<0$ \\
\cline { 2 - 5 } & $\mathrm{I}$ & $\mathrm{IV}$ & $\mathrm{I}$ & $\mathrm{III}$ \\
\hline
\end{tabular}

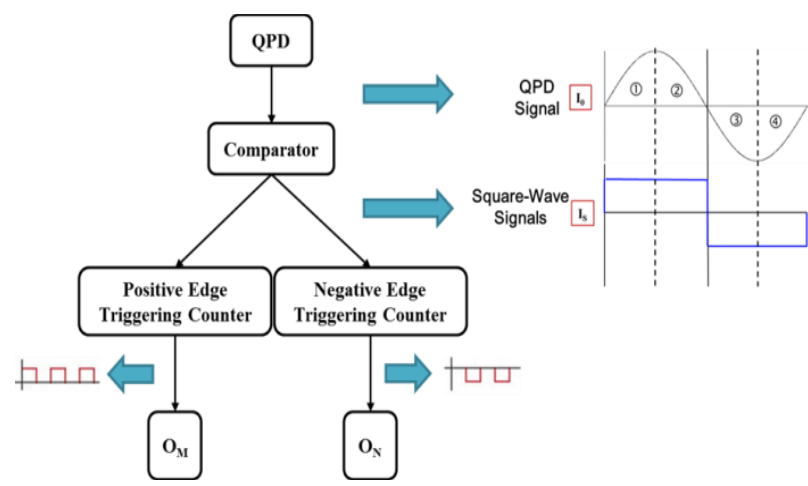

Figure 3. Scheme illustration of the corresponding signal output in measurement system. $O_{M}$ is the number of the positive edge triggering counting and $\mathrm{O}_{\mathrm{N}}$ is the number of the negative edge triggering counting.

In the last stage of the signal discriminant analysis procedure, we applied positive edge triggering counter and negative edge triggering counter as shown in Figure 3. Edge triggering is a trick to allow devices to create a very fine level trigger which is faster than all external feedback loops, allowing devices to accept inputs quickly, and then close off the entrance in time before their changing outputs will change the values of the inputs. The $\mathrm{O}_{M}$ and $\mathrm{O}_{N}$ are equal to the numbers of a halfwavelength long of the two-dimensional sinusoidal grating. Finally, having determined the $\mathrm{O}_{\mathrm{M}}, \mathrm{O}_{\mathrm{N}}$, initial positions, and terminal positions, the total displacements along the $\mathrm{X}$ - axis and $\mathrm{Z}$-axis can be extracted as follows:

$$
D_{S}=0.5 \times \mathrm{P}_{\mathrm{x}, \mathrm{z}} \times O_{M, N}+D_{I}+D_{T}
$$

Figure 4 shows a prototype sensor designed and built for achieving the measurement principleof the straightness error and displacement of a linear stage simultaneously. The size of the slope sensor is $86(\mathrm{~L}) \mathrm{x}$ $75(\mathrm{~W}) \times 50(\mathrm{H}) \mathrm{mm}$. As shown, the illumination collimated light (provided by a laser diode (Thorlab, Model: CPS532-C2)) is passed through a polarization beam-splitting device (Lambda, Model:OI-10.0BCQ442)and enters a focus lens(Thorlab, Model: LB1757) on the two-dimensional sinusoidal grating. It should be noticed that the polarization beam-splitting device which is disposed in the light path of the incident light and comprises a beam splitter (PBS) and a polarizer. The light reflected from the sample is passed through a polarizer and reflected on the mirror to enter the lens and the QPD (Ontrak, Model: PSM2-4Q).The two-dimensional signal of the QPD is captured using a real-time controller system (National Instruments, Model:cRIO-9024) with 16-bit resolution. The real-time controller system which comprises the reconfigurable FPGA chip (Xilinx Virtex-5) and A\&D (analog \& digital signal) input module is used to efficiently develop the electronic signal subdividing method for data acquisition and analysis.

In this study, we need to make an incident light from a light source disposed at a moving stage illuminating a two-dimensional sinusoidal grating disposed at a guiding rail, wherein a reflection light of the two-dimensional grating forms a light spot on a quadrant photodiode therefore making the quadrant photodiode generating a plurality of sensing signals. The position differences of the light spot are variable according to the movement of the stage thus further altering the voltage of the sensing signals of the quadrant photodiode. In performing the experiments, the prototype sensor and twodimensionalsinusoidal grating are set using precision linear air-bearing stage (Aerotech, Model: ABL1500) with a resolution of $1 \mathrm{~nm}$ as shown in Fig. 5 .

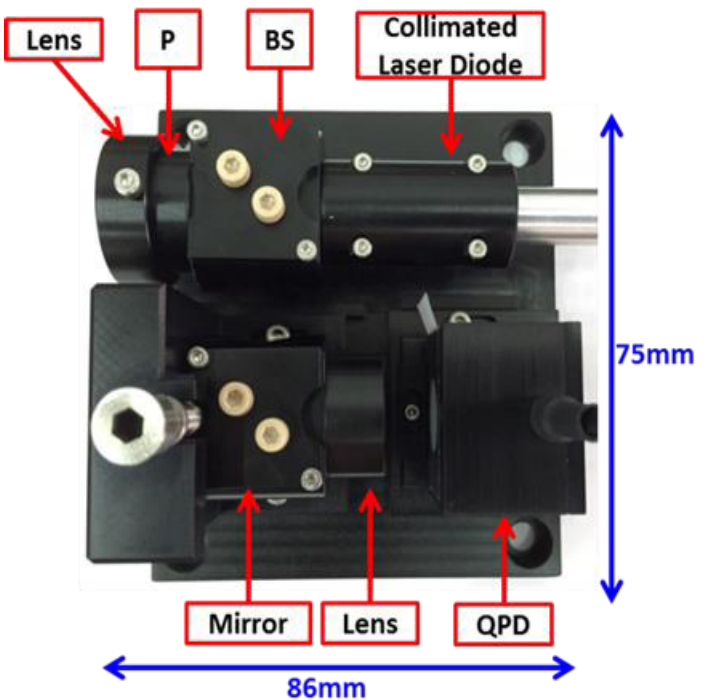

Figure 4. Photograph of the prototype sensor. P: polarizer, BS: beam splitter, and QPD: quadrant photo diodes. 


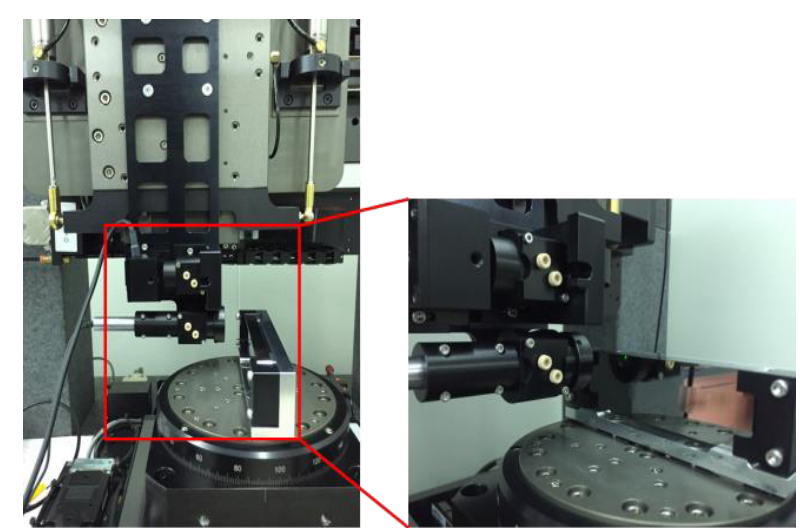

Figure 5. Photograph of the experimental setup.

On the other hand, the two dimensional sinusoidal structure on the stainless steel bar are machined by ultrasonic elliptical vibration cutting system. The two dimensional sinusoidal array on the stainless steel bar are machined by ultrasonic elliptical vibration cutting system. The stainless steel bar has a three dimensional microstructured surface, which is a superposition of periodic sinusoidal waves in the $\mathrm{X}$ - and Z-directions with spatial wavelengths of $350 \mu \mathrm{m}$ and amplitudes of $0.5 \mu \mathrm{m}$.

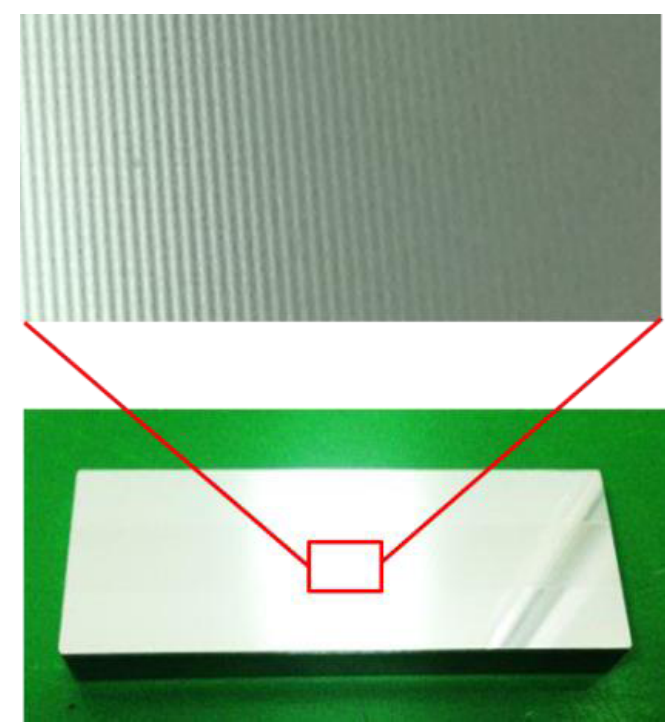

Figure 6. Photograph of the two dimensional sinusoidal grating.

\section{Experimental results}

The primary object of the study is to make an incident light from a light source disposed at a moving stage illuminating a two-dimensional sinusoidal grating disposed at a guiding rail, wherein a reflection light of the two-dimensional grating forms a light spot on a quadrant photodiode therefore making the quadrant photodiode generating a plurality of sensing signals. The position differences of the light spot are variable according to the movement of the stage thus further altering the intensity of the sensing signals of the quadrant photodiode.

Fig. 7 shows the three experimental results of sequence measurements in the $\mathrm{X}$-axis corresponding to the travel ranges $3.5 \mathrm{~mm}$, is extracted from the QPD along with position variance of the moving stage. The two dimensional sinusoidal grating was moved by a precision linear air-bearing stage along the $\mathrm{X}$-axis while the prototype sensor was kept stationary. As can be seen in the figure, the prototype sensor was able to measure the $\mathrm{X}$ - directional positions of the precision linear air-bearing stage by using the proposed phase-shift signal analysis method. The maximum repeatability error is $0.83 \mathrm{~nm}$. The extracted signals could be inserted into Eq. (6), Eq.(7), and Eq. (8) to calculate the straightness and displacement. The maximum error corresponding to the travel ranges $100 \mathrm{~mm}$ in the measured displacement is $0.393 \mathrm{~nm}$.

Fig. 8 shows the slope signal of sequency measurement corresponding to the travel ranges $3.5 \mathrm{~mm}$, is extracted from the QPD. It should be noticed that the blue datum lines of the figures are the $\mathrm{X}$-axis direction along with position variance of the moving stage. And the red datum lines of the figures are the vertical direction of the linear stage. Therefore, the $\mathrm{U}$ and $\mathrm{V}$ axis are corresponding to the horizontal direction and vertical direction of the QPD.

The experimental results show good verification with the instantaneous measurement method of the precision linear air-bearing stage.

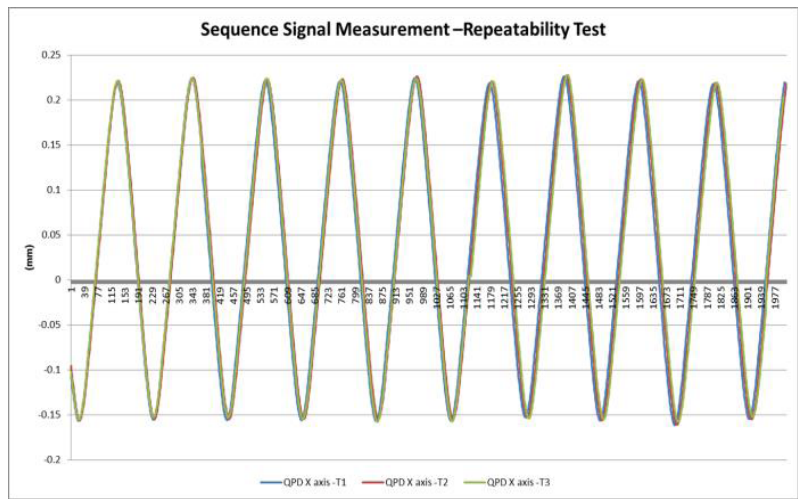

Figure 7. Sequence Signal Measurement -Repeatability Test of $\mathrm{X}$ axis.

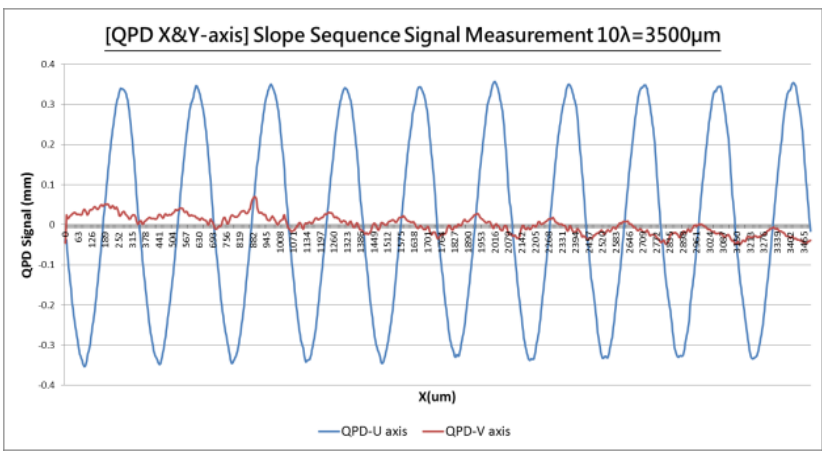

Figure 8. Slope Sequence Signal Measurement of the $\mathrm{X}$ and $\mathrm{Z}$ axis.

\section{Conclusion}

In this research, an electronic signal subdividing method is developed. This system is based on FPGA in combination with $\mathrm{A} / \mathrm{D}$ and $\mathrm{D} / \mathrm{A}$ converting circuits. Subdividing algorithm improves the segmenting signal amplitude method. Furthermore, we also construct a laser encoder for measuring straightness error and displacement of a linear stage simultaneously. The laser encoder consisting of the stainless steel bar and the sensor are developed for two-axis ( $\mathrm{X}$ - and $\mathrm{Z}$-axis) 
position measurement. Additionally, this methodology is especially suitable for linear stage of present invention is suitable to measure the displacement and the straightness error of the linear stage on line therefore instantaneously controlling the linear stage by information feedback. Moreover, the experimental results verified the instantaneous measurement method of the precision linear air-bearing stage.

\section{References}

1. A. H. Slocum, Precision machine design(Englewood Cliffs, NJ: Prentice-Hall, (1992)

2. W. Gao, Precision Nanometrology: Sensors and Measuring Systems for Nanomanufacturing, Springer, (2010)
3. J. G. Webster, The measurement, instrumentation, and sensors handbook, CRC Press LLC, (1999)

4. O. Riemer, Advances in ultraprecision manufacturing, Proc. Jpn. Soc. Precis. Eng., (2011)

5. C. Lee, G. H. Kim, S. K. Lee, Meas. Sci. Technol. 22, 105901 (2011)

6. C. Lee, G. H. Kim , S. K. Lee, Int. J. Prec. Manuf. Eng. 13, 9 (2012)

7. S. R. Park, T. K. Hoang, S. H. Yang, J. Mech. Sci. Technol. 24, 175 (2010)

8. C. B. Lee, S. K. Lee, J. Mech. Sci. Technol. 27, 141 (2013)

9. R.J. King, K.W. Raine, Opt. Eng. 201, 39 (1981)

10. C. Yin, W.Z. Chen, Opt. Eng. 304, 480 (1991)

11. S. Shimizu, H.S. Lee, N. Imai, Int. J. Japan Soc. Prec. Eng. 28, 273 (1994)

12. T.R. Armstrong, M.P. Fitzgerald, Measurement Sci., 1072 (1992) 